\title{
Cubic Rashba Effect in the Surface Spin Structure of Rare-Earth Ternary Materials
}

\author{
D. Yu. Usachov $\odot,{ }^{1}$ I. A. Nechaev $\odot,{ }^{2}$ G. Poelchen $\odot,{ }^{3}$ M. Güttler $\odot,{ }^{3}$ E. E. Krasovskii $\odot,{ }^{4,5,6}$ S. Schulz $\odot,{ }^{3}$ \\ A. Generalov $\odot,{ }^{7}$ K. Kliemt $\odot,{ }^{8}$ A. Kraiker $\odot,{ }^{8}$ C. Krellner $\odot,{ }^{8}$ K. Kummer, ${ }^{9}$ S. Danzenbächer, ${ }^{3}$ C. Laubschat, ${ }^{3}$ \\ A. P. Weber $\odot,{ }^{4}$ J. Sánchez-Barriga, ${ }^{10}$ E. V. Chulkov, ${ }^{1,4,5,11,12}$ A. F. Santander-Syro $\odot,{ }^{13}$ T. Imai $\odot,{ }^{14}$ K. Miyamoto, ${ }^{15}$ \\ T. Okuda๑, ${ }^{15}$ and D. V. Vyalikh $\oplus^{4,6, *}$ \\ ${ }^{1}$ St. Petersburg State University, 7/9 Universitetskaya Naberezhnaya, St. Petersburg, 199034, Russia \\ ${ }^{2}$ Department of Electricity and Electronics, FCT-ZTF, UPV-EHU, 48080 Bilbao, Spain \\ ${ }^{3}$ Institut für Festkörperphysik und Materialphysik, Technische Universität Dresden, D-01062 Dresden, Germany \\ ${ }^{4}$ Donostia International Physics Center (DIPC), 20018 Donostia/San Sebastián, Basque Country, Spain \\ ${ }^{5}$ Departamento de Física de Materiales UPV/EHU, 20080 Donostia/San Sebastián, Basque Country, Spain \\ ${ }^{6}$ IKERBASQUE, Basque Foundation for Science, 48013, Bilbao, Spain \\ ${ }^{7}$ Max IV Laboratory, Lund University, Box 118, 22100 Lund, Sweden \\ ${ }^{8}$ Kristall- und Materiallabor, Physikalisches Institut, Goethe-Universität Frankfurt, \\ Max-von-Laue Strasse 1, D-60438 Frankfurt am Main, Germany \\ ${ }^{9}$ European Synchrotron Radiation Facility, 71 Avenue des Martyrs, Grenoble, France \\ ${ }^{10}$ Helmholtz-Zentrum Berlin für Materialien und Energie, Elektronenspeicherring BESSY II, \\ Albert-Einstein-Strasse 15, D-12489 Berlin, Germany \\ ${ }^{11}$ Centro de Física de Materiales CFM-MPC and Centro Mixto CSIC-UPV/EHU, \\ 20018 Donostia/San Sebastián, Basque Country, Spain \\ ${ }^{12}$ Tomsk State University, Lenina Avenue 36, 634050, Tomsk, Russia \\ ${ }^{13}$ Université Paris-Saclay, CNRS, Institut des Sciences Moléculaires d'Orsay, 91405, Orsay, France \\ ${ }^{14}$ Graduate School of Science, Hiroshima University, 1-3-1 Kagamiyama, Higashi-Hiroshima 739-8526, Japan \\ ${ }^{15}$ Hiroshima Synchrotron Radiation Center, Hiroshima University, 2-313 Kagamiyama, Higashi-Hiroshima 739-0046, Japan
}

(Received 30 January 2020; revised manuscript received 13 April 2020; accepted 19 May 2020; published 12 June 2020)

\begin{abstract}
Spin-orbit interaction and structure inversion asymmetry in combination with magnetic ordering is a promising route to novel materials with highly mobile spin-polarized carriers at the surface. Spin-resolved measurements of the photoemission current from the Si-terminated surface of the antiferromagnet $\mathrm{TbRh}_{2} \mathrm{Si}_{2}$ and their analysis within an $a b$ initio one-step theory unveil an unusual triple winding of the electron spin along the fourfold-symmetric constant energy contours of the surface states. A two-band $\mathbf{k} \cdot \mathbf{p}$ model is presented that yields the triple winding as a cubic Rashba effect. The curious in-plane spinmomentum locking is remarkably robust and remains intact across a paramagnetic-antiferromagnetic transition in spite of spin-orbit interaction on $\mathrm{Rh}$ atoms being considerably weaker than the out-of-plane exchange field due to the $\mathrm{Tb} 4 f$ moments.
\end{abstract}

DOI: 10.1103/PhysRevLett.124.237202

Spin-orbit interaction (SOI) combined with exchange [1] or Kondo interactions [2] in noncentrosymmetric twodimensional (2D) systems is the basis for novel magnetic materials with spin-polarized carriers at the surface. The underlying phenomena are the momentum-dependent spinorbit splitting of 2D states governed by the Rashba effect and the Zeeman-like exchange splitting, whose simultaneous action brings about an exotic spin structure very different from textbook examples [1-4].

The Rashba effect significantly influences the spin properties of the carriers in magnets even when the exchange interaction is much stronger than SOI [3]. The SOI-induced splitting and in-plane spin-momentum locking often deviate from the prediction of the classical [linear, $\mathrm{R}^{(1)}$ ] Rashba model with its helical effective magnetic field (EMF). In reality, the $\mathbf{k}$ dependence of the splitting is far from linear, and the spin-momentum locking is not orthogonal $[5,6]$.

Vivid examples are semiconductor quantum wells $[7,8]$ and oxide surfaces and interfaces [9-13], which prominently feature the so-called cubic, $\mathrm{R}^{(3)}$, Rashba effect responsible for the nonlinear $\left(\alpha|\mathbf{k}|^{3}\right)$ dependence of the spin-orbit splitting of 2D heavy-hole states [14-16]. Remarkably, the EMF in the $\mathrm{R}^{(3)}$ model has a different symmetry from the $\mathrm{R}^{(1)}$ model, so that the in-plane pseudospin of the heavy hole rotates three times faster in moving around the Fermi contour and is no longer orthogonal to $\mathbf{k}$ everywhere [9,17-20].

Here, using spin- and angle-resolved photoelectron spectroscopy (SR-ARPES), we unveil a system that realizes a $\mathrm{R}^{(3)}$ effect for the true spin: the Si-terminated surface of 
the antiferromagnetic (AFM) $\mathrm{TbRh}_{2} \mathrm{Si}_{2}$ (TRS). It belongs to a family of $\mathrm{RET}_{2} \mathrm{Si}_{2}$ materials (RE and $\mathrm{T}$ are rare-earth and transition metal atoms, respectively) of the $\mathrm{ThCr}_{2} \mathrm{Si}_{2}$ type [21]. We report the first observation of the exotic spin structure predicted ab initio for the $\mathrm{RET}_{2} \mathrm{Si}_{2}$ family [3], which has the distinctive triple winding of the in-plane spin [1] along the fourfold-symmetric constant energy contour (CEC). In the present context, the term spin refers to the expectation value of the spin operator rather than to a pseudospin. We corroborate our observation with a calculation of the spin photocurrent within an ab initio one-step theory [22,23]. Furthermore, we derive from the full microscopic Hamiltonian a minimal relativistic $\mathbf{k} \cdot \mathbf{p}$ model that proves the observed spin structure to be due to the $\mathrm{R}^{(3)}$ effect.

The angle-resolved photoelectron spectroscopy (ARPES) experiments were performed at the I05 beam line of the Diamond Light Source, while the SR-ARPES measurements were conducted at the ESPRESSO instrument (BL9B) of the HiSOR facility [24] and at the SR-ARPES instrument of RGBL-2 beam line at BESSY-II. These instruments are equipped with VLEED and Mott detectors for spin analysis, respectively. All the data were measured at $\hbar \omega=55 \mathrm{eV}$ and linear polarization. Single crystalline samples of TRS were grown using a high-temperature indium-flux method [25]. The calculations in Fig. 1 were performed with the full-potential local orbital method [26] within density functional theory (DFT) (see Supplemental Material [27]). The ARPES spectra were calculated within the one-step theory [32], in which the outgoing electron is described by a (scalar-relativistic) time-reversed LEED state [33]. The initial states are eigenfunctions of a thick slab obtained from a large-size relativistic $\mathbf{k} \cdot \mathbf{p}$ Hamiltonian (see Supplemental Material [27]).

Antiferromagnetic TRS has the Néel temperature of $\sim 90 \mathrm{~K}$. In the AFM phase, the $\mathrm{Tb} 4 f$ moments in the $a b$ plane are ferromagnetically ordered with out-of-plane orientation [34]. The neighboring planes of Tb are separated by silicide $\mathrm{Si}-\mathrm{Rh}-\mathrm{Si}$ blocks and the ordered $\mathrm{Tb} 4 f$ moments couple antiferromagnetically along the $c$ axis. Upon cleavage, the resulting surface exhibits either $\mathrm{Tb}$ or $\mathrm{Si}$ termination. The latter has surface states in a large projected band gap around the $\bar{M}$ point. Figure 1(a) shows Fermi contours in PM (paramagnetic) and AFM phases. The surface states are seen as four-point stars around the corners of the surface Brillouin zone (SBZ) with a strong splitting typical of the AFM-ordered Si-terminated $\mathrm{RET}_{2} \mathrm{Si}_{2}$ materials [2,35-38].

The DFT band structure alongside the ARPES from the Si-terminated surface of TRS is shown for PM in Fig. 1(b) and for AFM in Fig. 1(c). The three surface states are labeled $\alpha, \beta$, and $\gamma$. The occupied states $\alpha$ and $\beta$ are clearly seen in the experiment in perfect agreement with the theory. For the PM phase, the states $\alpha, \beta$, and $\gamma$ have only in-plane spin components $S_{x}$ and $S_{y}$. The splitting obviously stems from the SOI of $\mathrm{Rh}$ in the noncentrosymmetric $\mathrm{Si}-\mathrm{Rh}-\mathrm{Si}-\mathrm{Tb}$ surface block $[2,3,38]$. The inset in the $\bar{M}-\bar{X}$ graph of Fig. 1(b) shows a magnified ARPES map of $\alpha$ with a well-resolved k-dependent splitting, which reaches $35 \mathrm{meV}$ at the Fermi level (see the black arrows). In


FIG. 1. (a) Fermi contours from ARPES for PM and AFM phases. Red and blue arrows indicate color schemes used for in-plane spin components derived from DFT calculation in graphs (b) and (c). Calculated and measured band structure for Si-terminated TRS for PM (b) and AFM (c) phases. The inset in (b) shows the spin-orbit splitting of the $\alpha$ state: black arrows indicate the 35 meV splitting. Black arrows in (c) indicate the $140 \mathrm{meV}$ splitting of the $\alpha$ state in AFM phase. Violet-brown palette shows calculated surface-projected bulk states. For the surface states the in-plane spin polarization is shown in red and blue, while yellow and green highlight the positive and negative spin component $S_{z}$, respectively. Spin-resolved ab initio CECs for the state $\alpha$ in the PM (d) and AFM (e) phase calculated at the binding energy of $0.23 \mathrm{eV}$. The in-plane spin orientation, $\mathbf{S}_{\|}$, is indicated by pink (purple) arrows for the inner (outer) contour. In AFM phase, the color of the spin vector $\mathbf{S}=\mathbf{S}_{\|}+S_{z} \hat{\mathbf{z}}$ shows the sign of $S_{z}$ as in Fig. 1(c). 
Figs. 1(b) and 1(c), the color indicates the orientation of the in-plane spin: clockwise (red) or anticlockwise (blue) assuming the origin at $\bar{M}$ [see the sketch of the SBZ in Fig. 1(a)]. Thus, the line $\bar{M}-\bar{\Gamma}$ is related to $\bar{M}-\bar{X}$ by an anticlockwise rotation around $\bar{M}$ by $\pi / 4$, whereby $\beta$ and $\gamma$ preserve their chirality (a classical Rashba behavior), whereas the chirality of $\alpha$ reverses: along $\bar{M}-\bar{\Gamma}$ the inner branch becomes red and the outer becomes blue [Fig. 1(c)] indicating the rotation of spin by $3 \pi / 4$.

The comparison of Figs. 1(b) and 1(c) suggests that the in-plane spin-momentum locking of the states $\alpha, \beta$, and $\gamma$ survives the transition from PM to AFM phase, i.e., the emergence of the ferromagnetic order within the Si-Rh-Si$\mathrm{Tb}$ surface block. The experimentally observed large Zeeman-like splitting [Fig. 1(c)] and the perfect symmetry of the split contours [Fig. 1(a)] points to the out-of-plane orientation of the Tb $4 f$ moments [38]. This gives rise to a sizable out-of-plane spin component $S_{z}$ of $\alpha$ and $\beta$ and strongly enhances their splitting, cf. Figs. 1(b) and 1(c), i.e., the exchange field felt by these states is much stronger than the SOI. The splitting of $\gamma$ is only slightly affected by the magnetization because of the negligible overlap with $\mathrm{Tb}$.

The calculated spin orientation for the state $\alpha$ over the CECs around $\bar{M}$ for both PM and AFM phases is shown in Figs. 1(d) and 1(e). For the PM phase, the spin lies in plane with a curious triple winding around the fourfoldsymmetric contours. In Fig. 1(d), the points between which the spin rotates by $2 \pi$ are shown by the encircled arrows. For the AFM phase, the sizable $S_{z}$ is due to the magnetization, Fig. 1(e). In spite of a relatively weak SOI on Rh atoms, the unique in-plane spin-momentum locking survives and remains practically unaltered in the AFM phase.

The essence of the triple winding is a fast rotation of the in-plane spin $\mathbf{S}_{\|}=S_{x} \hat{\mathbf{x}}+S_{y} \hat{\mathbf{y}}$ over a symmetry-irreducible fragment of CEC between the directions $\bar{M}-\bar{X}$ and $\bar{M}-\bar{\Gamma}$. Experimentally, we are limited by the fact that the spinquantization axes $X, Y$, and $Z$ of the spin analyzer depend on the emission direction $\mathbf{J}: X$ and $Y$ are perpendicular, and $Z$ is parallel to $\mathbf{J}$, the respective components of the photocurrent being $J_{X}, J_{Y}$, and $J_{Z}$. Thus, the $\mathbf{k}$ points must be selected such that the actual direction of $\mathbf{S}_{\|}$be close to $X$ or $Y$ axis and, at the same time, unambiguously manifest the effect. In particular, in Fig. 2(a), the energy distribution curves (EDCs) for the PM phase at two characteristic k points show the flip of the $J_{X}$ polarization, i.e., of the net-spin $X$ photocurrent.

In the AFM phase, for each branch of $\alpha$ or $\beta, S_{z}$ is large in magnitude and does not change sign along $\bar{X}-\bar{M}-\bar{X}$ and $\bar{\Gamma}-\bar{M}-\bar{\Gamma}$. This is expected to manifest itself in a sizable $J_{Z}$ polarization of the same sign (see Supplemental Material [27]). In the SR-ARPES measurements, depending on the tilt and polar angles, the large $S_{Z}$ also contributes to $J_{X}$ and $J_{Y}$. In turn, $J_{Z}$ may be influenced by $\mathbf{S}_{\|}$. An additional contribution to the $J_{Z}$ polarization may be caused by the
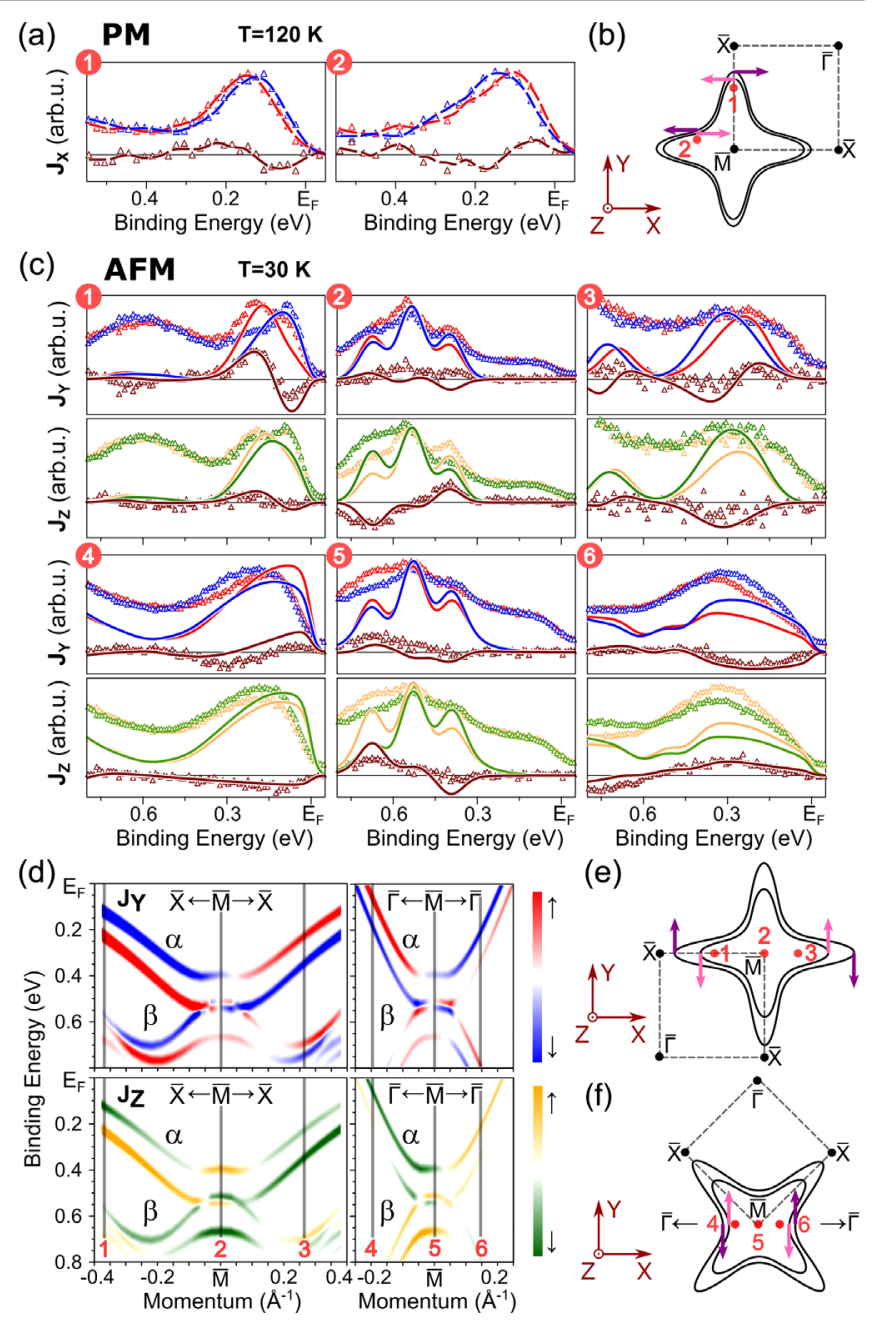

FIG. 2. SR-ARPES measurements of state $\alpha$ for PM (a) and AFM (c) phases of TRS. Measured $k$ points are denoted 1 and 2 for PM (b) and 1-6 for AFM (e)-(f). Red and blue symbols show spin-up $J^{\uparrow}$ and down $J^{\downarrow}$ EDCs for $J_{X}$ (a) and for $J_{Y}$ (c). Yellow and green symbols in (c) are $J_{Z}^{\uparrow}$ and $J_{Z}^{\downarrow}$. Brown symbols are the net spin $J^{\uparrow}-J^{\downarrow}$. Calculated EDCs for AFM (c) are shown with lines of the same color code. EDCs are energy broadened and $k$ averaged along the analyzer slit to mimic the experimental energy resolution and acceptance angle. Lines in graph (a) are a guide for the eye. Energy momentum maps in graph (d) show calculated net spin photocurrent without broadening and averaging. Theoretical data in (c) and (d) are averaged over the opposite magnetization directions. The $X Y Z$ axes of the analyzer relative to SBZ are shown in graphs (b), (e), and (f) by brown arrows (see Supplemental Material [27] for details of geometry). In (b), (e), and (f), arrows (pink for inner and purple for outer CEC) are a guide to relate EDCs in (a) and (c) to spin polarization in ground state.

light breaking the symmetry of the experiment. This is a matrix element effect (MEE), which depends on the light incidence and on the final state [23], and the present photoemission theory is instrumental in separating the different sources of $J_{Z}$. 
However, the observed behavior of $J_{Z}$ cannot be reconciled with the assumption that the signal comes from a single magnetic domain: $J_{Z}$ has opposite polarization in points 1 (4) and 3 (6), the $J_{Z}$ polarization is weak, and at $\bar{M}$ it changes sign depending on the experimental geometry, cf. points $2[\bar{X}-\bar{M}-\bar{X}$ setup, Fig. 2(e)] and $5[\bar{\Gamma}-\bar{M}-\bar{\Gamma}$ setup, Fig. 2(f)] in Fig. 2(c). According to our theory, Fig. 2(d), this implies a presence of oppositely magnetized domains (see Supplemental Material [27] for details). If we assume that in the ground state they counterbalance each other, then the averaging over magnetization would destroy the out-ofplane spin and eliminate its contribution to $J_{Z}$. This leads to the overall good agreement both at points 1 (4) and 3 (6) and at $\bar{M}$. Also, the $\mathbf{S}_{\|}$contribution to $J_{Z}$ turns out to be rather weak in the AFM phase, not only in the vicinity of $\bar{M}$ but also far from it. Thus, the $J_{Z}$ EDCs in Fig. 2(c) are a manifestation of the MEE, since the influence of the initialstate spin is here unimportant.

Another manifestation of the MEE is that over a rather wide range around $\bar{M}$ the $J_{Y}$ polarization strongly deviates from the spin $\mathbf{S}_{\|}$of the initial state, cf. Fig. 2(d) and 1(c), while sufficiently far from $\bar{M}$ it follows $\mathbf{S}_{\|}$, both in the theory and in the experiment. The good agreement between the theoretical and measured $J_{Y}$ and $J_{Z}$ in Fig. 2(c) fully supports the triple-winding interpretation of the measured spin structure.

To prove that the observed spin pattern is a manifestation of the $\mathrm{R}^{(3)}$ effect, let us focus on the PM phase. In the literature, the term "cubic" implies a specific form of the two-band Rashba effective Hamiltonian for the total angular momentum states $\left|j m_{j}\right\rangle$ with the $z$ projection $m_{j}=$ $\pm 3 / 2$ (in particular for 2D heavy holes) $[14,39,40]$. There, the cubic spin-orbit splitting of these states is due to the term $H_{\mathrm{R}}^{(3)}=i \tilde{\gamma}\left(k_{-}^{3} \sigma_{+}-k_{+}^{3} \sigma_{-}\right)$, where $k_{ \pm}=k_{x} \pm i k_{y}$, $\sigma_{ \pm}=\left(\sigma_{x} \pm i \sigma_{y}\right) / 2$, and $\sigma_{\rho}(\rho=x, y$, and $z)$ are Pauli matrices referring to a pseudospin since they operate on the $\left|j m_{j}\right\rangle$ states, as emphasized in Refs. [19,41]. To highlight the impact of the $\mathrm{R}^{(3)}$ effect on the pseudospin, the $\mathbf{k}$-cubic contribution is expressed as a Zeeman-like term $H_{\mathrm{R}}^{(3)}=$ $\tilde{\gamma} \boldsymbol{\sigma} \cdot \mathcal{B}_{\mathrm{R}}^{(3)}$, where $\mathcal{B}_{\mathrm{R}}^{(3)}=k^{3}\left(\sin 3 \varphi_{\mathbf{k}},-\cos 3 \varphi_{\mathbf{k}}, 0\right)$ is the EMF with $k=\sqrt{k_{x}^{2}+k_{y}^{2}}$ and $\varphi_{\mathbf{k}}$ being the polar angle of $\mathbf{k}$. Thus, the EMF drives pseudospin to be collinear with $\mathcal{B}_{\mathrm{R}}^{(3)}$ at a given $\mathbf{k}$ \{see Fig. 3(a)\} [15]. As a result, the triple winding of the pseudospins with a complete $2 \pi$ rotation of $\mathbf{k}$ is a hallmark of the cubic effect. In contrast, the $\mathrm{R}^{(1)}$ twoband Hamiltonian is $H_{\mathrm{R}}^{(1)}=i \tilde{\alpha}\left(k_{-} \sigma_{+}-k_{+} \sigma_{-}\right)=\tilde{\alpha} \boldsymbol{\sigma} \cdot \mathcal{B}_{\mathrm{R}}^{(1)}$ with a single winding of the EMF $\mathcal{B}_{\mathrm{R}}^{(1)}=k\left(\sin \varphi_{\mathbf{k}}\right.$, $\left.-\cos \varphi_{\mathbf{k}}, 0\right)$ [Fig. 3(a)].

In order to understand whether the observed triple winding of the true spin is a manifestation of the $\mathrm{R}^{(3)}$ effect, we draw on the microscopic approach of Refs. $[3,4,42]$ to derive a two-band $\mathbf{k} \cdot \mathbf{p}$ Hamiltonian of the form [27]

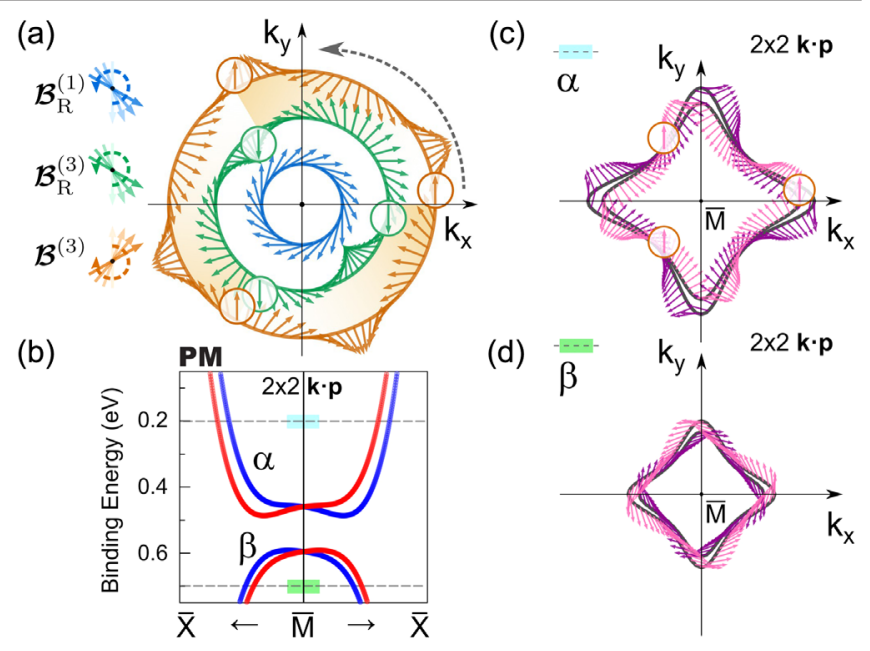

FIG. 3. (a) Effective spin-orbit magnetic fields $\mathcal{B}_{\mathrm{R}}^{(1)}\left(\mathrm{R}^{(1)}\right.$ effect, blue arrows), $\mathcal{B}_{\mathrm{R}}^{(3)}$ ("heavy-hole" $\mathrm{R}^{(3)}$ effect, green arrows), and $\mathcal{B}^{(3)}\left(\mathrm{R}^{(3)}\right.$ effect for the state $\alpha$, orange arrows) as a function of polar angle $\varphi_{\mathbf{k}}$. The legends on the left show the rotation direction of the vectors $\mathcal{B}_{\mathrm{R}}^{(1)}, \mathcal{B}_{\mathrm{R}}^{(3)}$, and $\mathcal{B}^{(3)}$ with the anticlockwise rotation of $\mathbf{k}$ (gray dashed arc arrow). (b) Band structure of the states $\alpha$ and $\beta$ by the Hamiltonian (1) presented by red $\left(S_{y}>0\right)$ and blue $\left(S_{y}<0\right)$ bands. Graphs (c) and (d) show spin-resolved CECs by the $\mathbf{k} \cdot \mathbf{p}$ model at the binding energy of 0.2 and $0.7 \mathrm{eV}$, respectively [indicated by dashed gray lines in graph (b)], with the $\mathbf{S}_{\|}$orientation shown by pink (purple) arrows for the inner contour and by purple (pink) for outer contour of the state $\alpha(\beta)$.

$$
\begin{aligned}
H_{\mathbf{k p}}^{2 \times 2}= & {\left[\epsilon+M(k) k^{2}+W\left(k_{+}^{4}+k_{-}^{4}\right)\right] \sigma_{0} } \\
& +i \tilde{\alpha}(k)\left(k_{-} \sigma_{+}-k_{+} \sigma_{-}\right)+i \tilde{\gamma}\left(k_{-}^{3} \sigma_{-}-k_{+}^{3} \sigma_{+}\right) .
\end{aligned}
$$

Here, $\epsilon$ is the surface-state energy at $\bar{M}$. The effective mass $M(k)=M^{(2)}+M^{(4)} k^{2}$ includes a second order correction, and $W$ describes the fourfold warping of the CECs. In Eq. (1), the parameter $\tilde{\alpha}(k)=\tilde{\alpha}^{(1)}+\tilde{\alpha}^{(3)} k^{2}$ accounts for the conventional (orthogonal) in-plane spin-momentum locking governed by $\mathcal{B}_{\mathrm{R}}^{(1)}$. The term with $\tilde{\gamma}$ is responsible for the $\mathrm{R}^{(3)}$ effect we are interested in. Note that the respective EMF $\mathcal{B}^{(3)}=k^{3}\left(\sin 3 \varphi_{\mathbf{k}}, \cos 3 \varphi_{\mathbf{k}}, 0\right)$ has a fourfold symmetry, whereas the "heavy-hole" field $\mathcal{B}_{\mathrm{R}}^{(3)}$ has a twofold symmetry [see Fig. 3(a)].

The spin polarization of the states $\alpha$ and $\beta$ rapidly grows in moving away from $\bar{M}$. Being practically unpolarized in the vicinity of $\bar{M}$, they become almost completely polarized far from it [Fig. 1(c)]. This is due to the interaction between $\alpha$ and $\beta$, and, naturally, the $2 \times 2$ Hamiltonian (1) does not have this property. However, if we associate the $\sigma$ matrices in Eq. (1) with the spin [27] [Fig. 3(b)], the Hamiltonian turns out to accurately describe both the triple winding in the $\alpha$ contour [Fig. 3(c)] and the single winding in the $\beta$ contour [Fig. 3(d)]. 
TABLE I. Parameters of the Hamiltonian (1) for the states $\alpha$ and $\beta$ in Rydberg atomic units: $\hbar=2 m_{0}=e^{2} / 2=1$.

\begin{tabular}{lcc}
\hline \hline Surface state & $\alpha$ & $\beta$ \\
\hline$\epsilon / M^{(2)}$ & $-0.035 /-1.55$ & $-0.045 /-0.96$ \\
$M^{(4)} / W$ & $3.9 \times 10^{3} /-1.4 \times 10^{3}$ & $-3.9 \times 10^{3} / 1.4 \times 10^{3}$ \\
$\tilde{\alpha}^{(1)} / \tilde{\alpha}^{(3)} / \tilde{\gamma}$ & $0.039 /-11.85 /-23.35$ & $0.039 / 6.05 / 3.30$ \\
\hline \hline
\end{tabular}

The spin of the state $\alpha$ follows the winding of $\boldsymbol{B}^{(3)}$ [Fig. 3(c)], revealing the dominance of the $k_{ \pm}$-cubic term over the $k_{ \pm}$-linear one. Actually, for this state $\tilde{\gamma}$ is almost twice as large as $\tilde{\alpha}^{(3)}$ (the microscopically obtained parameters are listed in Table I). By contrast, for the state $\beta$, the $k_{ \pm}$-linear term dominates because here $\tilde{\gamma}$ is two times smaller than $\tilde{\alpha}^{(3)}$ (Table I). This results in a single spin winding around the CEC [Fig. 3(d)]. Thus, we conclude that the observed spin structure is an interference of the $\mathrm{R}^{(1)}$ and $\mathrm{R}^{(3)}$ effects that yields the winding of surface-state spin in Fig. 1(d).

In summary, with SR-ARPES we unveiled an unusual inplane spin structure of surface states with a triple winding of the spin along the constant energy contours in the exemplary antiferromagnet $\mathrm{TbRh}_{2} \mathrm{Si}_{2}$, a representative of a wide class of rare-earth ternary materials. The unique spin structure appears to be rather robust and persists when a strong out-of-plane magnetic order of the $\mathrm{Tb}$ moments sets in. This property is due to a cubic Rashba effect, whose strength can be tuned by a proper choice of the transition metal atom. Combined with a different orientation and strength of the exchange field near the surface, it can create a variety of fascinating spin patterns. A fundamentally important finding is that relatively light atoms like $\mathrm{Rh}$ may give rise to a distinct spin-momentum gyration stable in an exchange field much stronger than the spinorbit field.

This work was supported by the German Research Foundation (Grants No. KR-3831/5-1, No. LA655/20-1, GRK1621, Fermi-NESt No. ANR-16-CE92-0018, and SFB1143, project-id 247310070) and the Spanish Ministry of Science, Innovation, and Universities (Grant Nos. FIS2016-76617-P and MAT-2017-88374-P). We also acknowledge funding from the Department of Education of the Basque government (Grant No. IT1164-19), St. Petersburg State University (Project ID 51126254), and the Russian Foundation for Basic Research (Grant No. 2032-70127). The SR-ARPES experiments at HiSOR were performed with the approval of the Proposal Assessing Committee of the Hiroshima Synchrotron Radiation Center (Proposal No. 18BG023). We also acknowledge the Impuls-und Vernetzungsfonds der Helmholtz Gemeinschaft (Grant No. HRSF-0067).
*Corresponding author.

Denis.Vyalikh@dipc.org

[1] S. Schulz, I. A. Nechaev, M. Güttler, G. Poelchen, A. Generalov, S. Danzenbächer, A. Chikina, S. Seiro, K. Kliemt, A. Yu. Vyazovskaya, T. K. Kim, P. Dudin, E. V. Chulkov, C. Laubschat, E. E. Krasovskii, C. Geibel, C. Krellner, K. Kummer, and D. V. Vyalikh, Emerging 2Dferromagnetism and strong spin-orbit coupling at the surface of valence-fluctuating $\mathrm{EuIr}_{2} \mathrm{Si}_{2}$, npj Quantum Mater. 4, 26 (2019).

[2] A. Generalov, J. Falke, I. A. Nechaev, M. M. Otrokov, M. Güttler, A. Chikina, K. Kliemt, S. Seiro, K. Kummer, S. Danzenbächer, D. Usachov, T. K. Kim, P. Dudin, E. V. Chulkov, C. Laubschat, C. Geibel, C. Krellner, and D. V. Vyalikh, Strong spin-orbit coupling in the noncentrosymmetric Kondo lattice, Phys. Rev. B 98, 115157 (2018).

[3] I. A. Nechaev and E. E. Krasovskii, Relativistic splitting of surface states at Si-terminated surfaces of the layered intermetallic compounds $R T_{2} \mathrm{Si}_{2}(R=$ rare earth; $T=\mathrm{Ir}$, Rh), Phys. Rev. B 98, 245415 (2018).

[4] I. A. Nechaev and E. E. Krasovskii, Spin polarization by first-principles relativistic $\mathrm{k} \cdot \mathrm{p}$ theory: Application to the surface alloys $\mathrm{PbAg}_{2}$ and $\mathrm{BiAg}_{2}$, Phys. Rev. B 100, 115432 (2019).

[5] L. V. Bondarenko, D. V. Gruznev, A. A. Yakovlev, A. Y. Tupchaya, D. Usachov, O. Vilkov, A. Fedorov, D. V. Vyalikh, S. V. Eremeev, E. V. Chulkov, A. V. Zotov, and A. A. Saranin, Large spin splitting of metallic surface-state bands at adsorbate-modified gold/silicon surfaces, Sci. Rep. 3, 1826 (2013).

[6] R. Friedrich, V. Caciuc, G. Bihlmayer, N. Atodiresei, and S. Blügel, Designing the Rashba spin texture by adsorption of inorganic molecules, New J. Phys. 19, 043017 (2017).

[7] R. Moriya, K. Sawano, Y. Hoshi, S. Masubuchi, Y. Shiraki, A. Wild, C. Neumann, G. Abstreiter, D. Bougeard, T. Koga, and T. Machida, Cubic Rashba Spin-Orbit Interaction of a Two-Dimensional Hole Gas in a Strained-Ge/SiGe Quantum Well, Phys. Rev. Lett. 113, 086601 (2014).

[8] H. Liu, E. Marcellina, A. R. Hamilton, and D. Culcer, Strong Spin-Orbit Contribution to the Hall Coefficient of Two-Dimensional Hole Systems, Phys. Rev. Lett. 121, 087701 (2018).

[9] H. Nakamura, T. Koga, and T. Kimura, Experimental Evidence of Cubic Rashba Effect in an Inversion-Symmetric Oxide, Phys. Rev. Lett. 108, 206601 (2012).

[10] P. D. C. King, S. M. Walker, A. Tamai, A. de la Torre, T. Eknapakul, P. Buaphet, S.-K. Mo, W. Meevasana, M. S. Bahramy, and F. Baumberger, Quasiparticle dynamics and spin-orbital texture of the $\mathrm{SrTiO}_{3}$ two-dimensional electron gas, Nat. Commun. 5, 3414 (2014).

[11] J. Varignon, L. Vila, A. Barthélémy, and M. Bibes, A new spin for oxide interfaces, Nat. Phys. 14, 322 (2018).

[12] S. Gariglio, A. D. Caviglia, J.-M. Triscone, and M. Gabay, A spin-orbit playground: surfaces and interfaces of transition metal oxides, Rep. Prog. Phys. 82, 012501 (2018).

[13] W. Lin, L. Li, F. Doğan, C. Li, H. Rotella, X. Yu, B. Zhang, Y. Li, W. S. Lew, S. Wang, W. Prellier, S. J. Pennycook, J. Chen, Z. Zhong, A. Manchon, and T. Wu, Interface-based tuning of Rashba spin-orbit interaction in asymmetric oxide 
heterostructures with $3 d$ electrons, Nat. Commun. 10, 3052 (2019).

[14] L. G. Gerchikov and A. V. Subashiev, Spin splitting of sizequantization subbands in asymmetric heterostructures, Sov. Phys. Semicond. 26, 73 (1992).

[15] R. Winkler, Spin-Orbit Coupling Effects in TwoDimensional Electron and Hole Systems (Springer, Berlin, 2003).

[16] E. Marcellina, A. R. Hamilton, R. Winkler, and D. Culcer, Spin-orbit interactions in inversion-asymmetric twodimensional hole systems: A variational analysis, Phys. Rev. B 95, 075305 (2017).

[17] L. Wang and M. W. Wu, Hole spin relaxation in $p$-type (111) GaAs quantum wells, Phys. Rev. B 85, 235308 (2012).

[18] K. Kondo, Spin filter effects in an Aharonov-Bohm ring with double quantum dots under general Rashba spin-orbit interactions, New J. Phys. 18, 013002 (2015).

[19] S. Bladwell and O.P. Sushkov, Magnetic focusing of electrons and holes in the presence of spin-orbit interactions, Phys. Rev. B 92, 235416 (2015).

[20] K. V. Shanavas, Theoretical study of the cubic Rashba effect at the $\mathrm{SrTiO}_{3}(001)$ surfaces, Phys. Rev. B 93, 045108 (2016).

[21] I. Felner and I. Nowik, Itinerant and local magnetism, superconductivity and mixed valency phenomena in $R M_{2} \mathrm{Si}_{2},(R=$ rare earth, $M=\mathrm{Rh}, \mathrm{Ru}), \mathrm{J}$. Phys. Chem. Solids 45, 419 (1984).

[22] A. Kimura, E. E. Krasovskii, R. Nishimura, K. Miyamoto, T. Kadono, K. Kanomaru, E. V. Chulkov, G. Bihlmayer, K. Shimada, H. Namatame, and M. Taniguchi, Strong RashbaType Spin Polarization of the Photocurrent from Bulk Continuum States: Experiment and Theory for Bi(111), Phys. Rev. Lett. 105, 076804 (2010).

[23] H. Bentmann, H. Maaß, E. E. Krasovskii, T. R. F. Peixoto, C. Seibel, M. Leandersson, T. Balasubramanian, and F. Reinert, Strong Linear Dichroism in Spin-Polarized Photoemission from Spin-Orbit-Coupled Surface States, Phys. Rev. Lett. 119, 106401 (2017).

[24] T. Okuda, K. Miyamaoto, H. Miyahara, K. Kuroda, A. Kimura, H. Namatame, and M. Taniguchi, Efficient spin resolved spectroscopy observation machine at Hiroshima Synchrotron Radiation Center, Rev. Sci. Instrum. 82, 103302 (2011).

[25] K. Kliemt, M. Peters, F. Feldmann, A. Kraiker, D.-M. Tran, S. Rongstock, J. Hellwig, S. Witt, M. Bolte, and C. Krellner, Crystal growth of materials with the $\mathrm{ThCr}_{2} \mathrm{Si}_{2}$ structure type, Cryst. Res. Technol. 55, 1900116 (2019).

[26] K. Koepernik and H. Eschrig, Full-potential nonorthogonal local-orbital minimum-basis band-structure scheme, Phys. Rev. B 59, 1743 (1999).

[27] See Supplemental Material at http://link.aps.org/ supplemental/10.1103/PhysRevLett.124.237202 for the geometry of SR-ARPES experiments, the details of the DFT calculations, the calculations of spin photocurrent from magnetic surfaces based on a large-size relativistic $\mathbf{k} \cdot \mathbf{p}$
Hamiltonian, and the derivation of four- and two-band relativistic $\mathbf{k} \cdot \mathbf{p}$ Hamiltonians up to the third and fourth order in $\mathbf{k}$, including the additional Refs. [28-31].

[28] P.-O. Löwdin, A note on the quantum-mechanical perturbation theory, J. Chem. Phys. 19, 1396 (1951).

[29] J. R. Schrieffer and P. A. Wolff, Relation between the Anderson and Kondo Hamiltonians, Phys. Rev. 149, 491 (1966).

[30] E. E. Krasovskii, Accuracy and convergence properties of the extended linear augmented-plane-wave method, Phys. Rev. B 56, 12866 (1997).

[31] E. E. Krasovskii, F. Starrost, and W. Schattke, Augmented Fourier components method for constructing the crystal potential in self-consistent band-structure calculations, Phys. Rev. B 59, 10504 (1999).

[32] I. Adawi, Theory of the surface photoelectric effect for one and two photons, Phys. Rev. 134, A788 (1964).

[33] E. E. Krasovskii and W. Schattke, Calculation of the wave functions for semi-infinite crystals with linear methods of band theory, Phys. Rev. B 59, R15609 (1999).

[34] S. Quezel, J. Rossat-Mignod, B. Chevalier, P. Lejay, and J. Etourneau, Magnetic ordering in $\mathrm{TbRh}_{2} \mathrm{Si}_{2}$ and $\mathrm{CeRh}_{2} \mathrm{Si}_{2}$, Solid State Commun. 49, 685 (1984).

[35] A. Chikina, M. Höppner, S. Seiro, K. Kummer, S. Danzenbächer, S. Patil, A. Generalov, M. Güttler, Yu. Kucherenko, E. V. Chulkov, Yu. M. Koroteev, K. Koepernik, C. Geibel, M. Shi, M. Radovic, C. Laubschat, and D. V. Vyalikh, Strong ferromagnetism at the surface of an antiferromagnet caused by buried magnetic moments, Nat. Commun. 5, 3171 (2014).

[36] M. Güttler, K. Kummer, S. Patil, M. Höppner, A. Hannaske, S. Danzenbächer, M. Shi, M. Radovic, E. Rienks, C. Laubschat, C. Geibel, and D. V. Vyalikh, Tracing the localization of $4 f$ electrons: Angle-resolved photoemission on $\mathrm{YbCo}_{2} \mathrm{Si}_{2}$, the stable trivalent counterpart of the heavyfermion $\mathrm{YbRh}_{2} \mathrm{Si}_{2}$, Phys. Rev. B 90, 195138 (2014).

[37] M. Güttler et al., Robust and tunable itinerant ferromagnetism at the silicon surface of the antiferromagnet $\mathrm{GdRh}_{2} \mathrm{Si}_{2}$, Sci. Rep. 6, 24254 (2016).

[38] A. Generalov et al., Spin orientation of two-dimensional electrons driven by temperature-tunable competition of spin-orbit and exchange-magnetic interactions, Nano Lett. 17, 811 (2017).

[39] R. Winkler, Rashba spin splitting in two-dimensional electron and hole systems, Phys. Rev. B 62, 4245 (2000).

[40] R. Winkler, D. Culcer, S. J. Papadakis, B. Habib, and M. Shayegan, Spin orientation of holes in quantum wells, Semicond. Sci. Technol. 23, 114017 (2008).

[41] J. Schliemann and D. Loss, Spin-Hall transport of heavy holes in III-V semiconductor quantum wells, Phys. Rev. B 71, 085308 (2005).

[42] I. A. Nechaev and E. E. Krasovskii, Relativistic k·p hamiltonians for centrosymmetric topological insulators from ab initio wave functions, Phys. Rev. B 94, 201410(R) (2016). 